\title{
TERAPEUTICA
}

\section{La terapia de reemplazo hormonal es beneficiosa en la mayoría de las mujeres}

Col NF, Eckman MH, Karas RH et al. Patient-Specific Decisions About Hormone Replacement Therapy in Postmenopausal Women. JAMA. 1997; 277:1140-47.

\section{Objetivo}

Evaluar el efecto de la TRH en la expectativa de vida de mujeres postmenopáusicas con distintos perfiles de riesgo para enfermedad coronaria, cáncer de mama y fractura de cadera.

\section{Diseño}

Análisis de decisión utilizando un modelo matemático. Se utilizaron modelos de regresión publicados para estimar la incidencia de enfermedad coronaria, cáncer de mama, fractura de cadera y cáncer de endometrio, y su impacto en la expectativa de vida según factores de riesgo. El impacto de la terapia hormonal en la incidencia de las distintas enfermedades fue estimado de estudios epidemiológicos publicados.

\section{Lugar}

Modelo matemático aplicable a la atención primaria

\section{Intervención}

Terapia de reemplazo hormonal versus no tratamiento

\section{Medición de Resultados}

Expectativa de vida en las cohortes del modelo

\section{Resultados}

La terapia de reemplazo hormonal aumentaría la expectativa de vida en casi todas las mujeres postmenopáusicas, y en algunos subgrupos hasta más de tres años.
Esto depende principalmente de los factores de riesgo individuales para enfermedad coronaria y cáncer de mama. Por ejemplo: mujeres sin factores de riesgo cardiovascular ni para osteoporosis aumentarían su expectativa de vida menos de dos meses; en cambio aquellas con factores de riesgo para ambas patologías la aumentarían más de tres años. Para las mujeres con al menos un factor de riesgo para enfermedad coronaria, la hormonoterapia extendería la expectativa de vida. Aquellas sin factores de riesgo coronarios ni de fractura de cadera pero con dos familiares de primer grado con cáncer de mama no deberían recibir hormonoterapia de reemplazo.

\section{Conclusiones}

El beneficio de la terapia de reemplazo hormonal para reducir la probabilidad de desarrollar enfermedad coronaria parece superar los riesgos de cáncer de mama para casi todas las mujeres en que se considera este tratamiento. Este estudio sugiere que la terapia de reemplazo hormonal debería ser más ampliamente indicada.

Apoyo Económico: en parte National Library of Medicine y Agency for Health Care Policy and Research, USA

\section{Comentario}

Para ofrecer tratamientos preventivos la evidencia requerida es aún más importante que para tratamientos de personas enfermas, ya que estamos tratando a mujeres sanas. Actualmente está en curso un gran estudio randomizado (Women Health Initiative) evaluando el efecto de la TRH en la mortalidad en pacientes postmenopáusicas, pero recién en la próxima década tendremos novedades. El estudio prospectivo randomizado es el mejor medio para sacar conclusiones válidas en intervenciones terapéuticas, ya que la intervención se realiza al azar y así se eliminan distintos sesgos que son inherentes a cualquier estudio no randomizado. Hasta tanto no haya evidencia grado I (estudios randomizados), es necesario recurrir a estudios como los que revisamos en este número de Evidencia. Estos dos estudios con distinto diseño llegan a conclusiones similares con respecto a la TRH. El estudio de Grodstein es un análisis del estudio de las enfermeras, una cohorte prospectiva de 121.700 enfermeras contactadas en 1976 seguidas hasta la fecha con cuestionarios cada dos años (1). Este diseño es muy poderoso para establecer asociaciones por ejemplo entre utilización de TRH y mortalidad. El hallazgo principal, la disminución de la mortalidad global en las que comenzaron recientemente con TRH, es similar a otras investigaciones. Este beneficio se obtiene por una reducción dramática de las muertes coronarias y una disminución menor de la mortalidad por cáncer. También demuestran que la reducción de la mortalidad cardiovascular no se debe a la prescripción selectiva de estrógenos a mujeres libres de enfermedad o a la suspensión del tratamiento al inicio de una enfermedad fatal. Una gran ventaja de este estudio es que evalúa la mortalidad en relación tanto a la duración de la TRH como a cuán reciente fue ésta. El efecto protector de la TRH se pierde a los cinco años de suspenderla, y la utilización por más de 10 años no provee beneficios adicionales a quienes recibían TRH en ese momento. El beneficio disminuyó luego de diez años debido principalmente a la mayor mortalidad por cáncer de mama en este grupo (hay que tener en cuenta que la proporción de muertes por cáncer de mama en este estudio fue mucho mayor que en la población general, lo que limita la generalización de los resultados). La mortalidad por cáncer de mama se redujo en general en la población, pero la mayor mortalidad en quienes continuaban con TRH por más de diez años es un hallazgo preocupante, sobre todo cuando hay evidencia del aumento de la incidencia de cáncer de mama en utilizadoras a largo plazo (23). Un punto importante es que las mujeres con antecedentes familiares de cáncer de mama que utilizan TRH no tienen mayor riesgo de muerte que las que no tienen historia familiar. 